\title{
Autosomal recessive gingival hyperplasia and dental anomalies caused by a 29-base pair duplication in the FAM20A gene
}

\author{
Journal of Human Genetics (2013) 58, 566-567; doi:10.1038/jhg.2013.44; published online 23 May 2013
}

Amelogenesis imperfecta (AI; MIM 104530) is a heterogeneous collection of disorders encompassing inherited congenital defects of dental enamel formation, causing variably small, discolored, pitted, grooved, or fragile primary or permanent teeth. At least 14 syndromic and non-syndromic AI subtypes have been described, including autosomal dominant and recessive, and $\mathrm{X}$-linked recessive. Gene mutations that underlie both the non-syndromic forms and the tricho-dento-osseous

(MIM

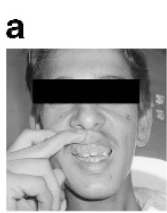

b

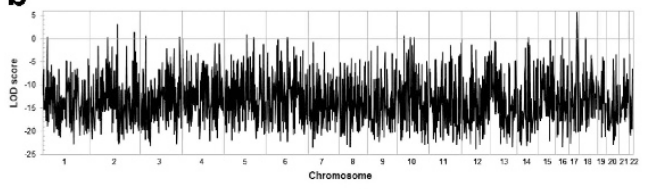

c

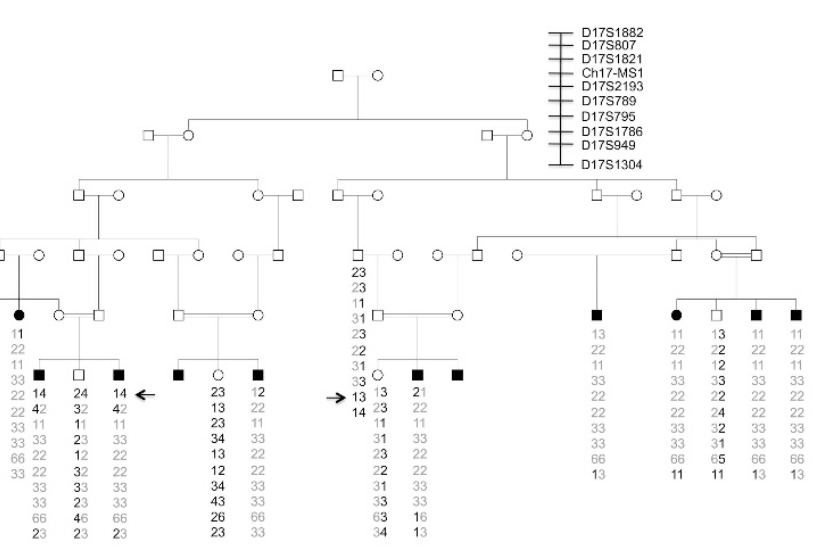

d
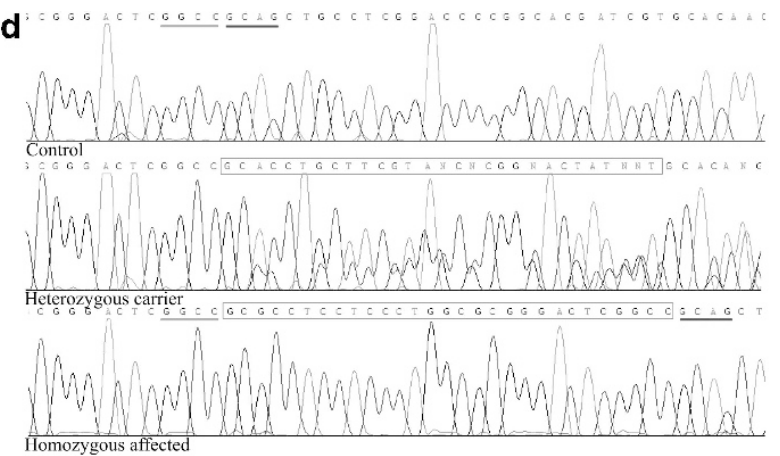

e atgocogggotgcgocgggaaccgcctactgactetgctgctgctg

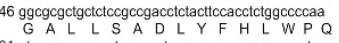
91 gtacagcoccagctgcggcctcoggagcgoccogcgggggtgcocg

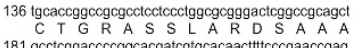
181 gcctcggaccccggcacgatcotgcacaactttlcccgaaccogag

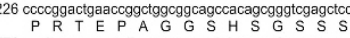

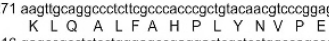
316 gagccogctctctctgggagccgaggactcogctctggocagccag

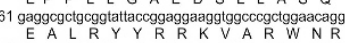
cgacacaagatgtacagagagcagatg 4 A

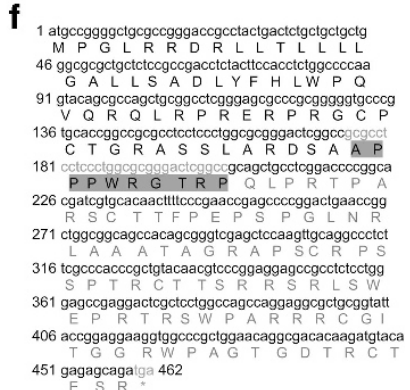

g

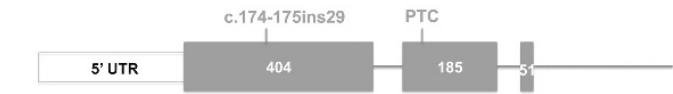

Figure 1 Homozygous 29-bp duplication in the FAM2OA gene, in a Pakistani family with gingival hyperplasia and dental anomalies. (a) Clinical phenotype of an affected individual reveals teeth abnormalities and gingival hyperplasia. (b) Autozygosity mapping using the low-density Affymetrix $10 \mathrm{~K}$ genotyping array. A maximum LOD score of 5.63 was obtained for an 11-Mb region on chromosome 17. (c) Pedigree and haplotype analysis. Microsatellite markers spanning the $11-\mathrm{Mb}$ region of homozygosity were used to genotype 16 family members and allowed narrowing of the linkage interval to $3.69 \mathrm{Mb}$ on chromosome 17 . Arrows indicate the key recombination events between markers D17S807 and D17S1821, and D17S1786 and D17S949. (d) Sanger sequencing identifies a homozygous 29-bp duplication in the first exon of the FAM20A gene, c.174-175ins29, in affected individuals. Representative electropherograms for control, heterozygous carrier and homozygous affected individuals are shown (red box shows inserted nucleotides, $5^{\prime}$ and $3^{\prime}$ nucleotides flanking the insertion are underlined in blue and green, respectively). (e and f) Translation of wild-type (e) and mutant (f) FAM2OA transcript. For simplicity, only part of the wild-type protein sequence is shown in e If translated, the mutant FAM20A protein would contain 10 additional amino acids (highlighted in red) followed by 85 foreign amino acids (in blue) and a PTC in exon 2. Owing to its nature, c.174-175ins29 is likely to result in activation of the Nonsense mediated mRNA decay (NMD) pathway and degradation of the mutant transcript prior to translation. (g) Schematic diagram of exons $1-3$ of the FAM20A gene showing the c.174175 ins 29 duplication and PTC in exon 2, located $257 \mathrm{bp}$ downstream of the mutation site and $1164 \mathrm{bp}$ upstream of the wild-type termination codon within the mRNA sequence. A full color version of this figure is available at the Journal of Human Genetics journal online. 
190320) and Jalili syndromes (MIM 217080) have recently been described. ${ }^{1}$

We studied a large consanguineous Pakistani family in which all members had severe gingival hyperplasia and dental abnormalities, including delayed tooth eruption or failure of tooth development (Figure 1a). On examination, the adults had primarily gingival disease, while children had prominent dental abnormalities with minimal, possibly secondary, gingival hyperplasia. Many family members could not chew hard food, because their gingiva covered molar and pre-molar teeth. None of the family members had any renal abnormalities.

After obtaining informed consent, we undertook a genome-wide scan on 16 family members using the Affymetrix $10 \mathrm{~K}$ SNP. Haplotypes, or clusters of SNPs that tend to be inherited together, were inferred from the data by Genespring GT (Agilent Software). Initial analysis included genome-wide autozygosity mapping to identify regions identical by descent that were shared among affected individuals, and parametric linkage analysis assuming a recessive mode of inheritance with $100 \%$ penetrance and a disease allele frequency of 0.001 . We identified an $11-\mathrm{Mb}$ region of excess homozygozity shared among affected individuals on chromosome 17 (autozygozity logarithm of the odds $(\mathrm{LOD})=6.53)$. Microsatellite markers spanning this region were then used to genotype all family members. Key recombination events were detected between markers D17S807 and D17S1821, and D17S1786 and D17S949 (Figure 1b), which allowed the linkage interval to be narrowed to $3.69 \mathrm{Mb}$. This region contains 34 genes.

Homozygous mutations in the gene FAM20A have recently been shown to cause a phenotype similar to that observed in our family. ${ }^{2,3}$ As FAM20A was included in our linkage interval, we used a candidate gene approach; we PCR amplified and directly sequenced all 11 exons of FAM20A (ABI Prism 310 Automated Sequencer). We identified a homozygous 29-bp duplication in exon 1, c.174-175ins29, which causes a frameshift and a premature termination codon (PTC) in exon 2,257-bp downstream of the mutation site (Figures $1 \mathrm{~d}-\mathrm{g}$ ). The mutation occurs between the first and second nucleotides of codon 59 (A, arginine), resulting in the potential insertion of 10 amino acids (APPPWRGTRP) followed by 85 foreign amino acids (Figures le and f). However, given the location of the PTC, this mutation likely activates nonsense mRNAmediated decay, an mRNA surveillance pathway that promotes degradation of mRNAs containing PTCs, preventing the synthesis of truncated or potentially harmful proteins. Consequently, the levels of FAM20A protein in the homozygous affected individuals are expected to be markedly reduced or absent. The c.174-175ins29 duplication, which was excluded from 100 unrelated control Pakistani individuals by direct sequencing, completely co-segregated with the disease phenotype. This is the first reported duplication in the FAM20A gene.

FAM20A belongs to a family of secreted proteins (FAM20A, FAM20B, FAM20C), which regulates differentiation of hematopoietic cells and bone development., Within the mouse incisor tooth, FAM20A mRNA and protein are expressed in the secretory and maturation stages of the ameloblasts, the suprabasal layers of the gingival epithelium, and the odontoblasts, a localization pattern consistent with the phenotype of AI and gingival hyperplasia. ${ }^{2}$ Vogel et al. ${ }^{6}$ studied knock-out mice for FAM20A and FAM20C, and identified that mice which were FAM20A $(-/-)$ and FAM20C $(-/-)$ had severe AI. Additionally, FAM20A $(-/-)$ mice had disseminated intrapulmonary and intra-arterial calcifications, raising the possibility of systemic disease in patients with FAM20A mutations.

Although further mechanistic studies are required to investigate the molecular function of FAM20A, published data support its crucial role for enamel development and gingival homeostasis. We have identified the first duplication in FAM20A and the fifth independent mutation associated with gingival hyperplasia and dental anomalies.

\section{ACKNOWLEDGEMENTS}

We are grateful to the family members for their participation in this study. We would like to thank Dr Munenari Itoh for his valuable discussion, comments, and ideas, and H Lam and M Zhang for technical assistance. We appreciate the collaboration with Drs C Higgins, $\mathrm{C}$ Luke and other members of the Christiano laboratory. This study was supported in part by USPHS/NIH grant RO1AR44924 from NIAMS (to AMC).

Author Contributions: RMC and MK carried out the molecular genetic studies, sequence alignments and preparation of the manuscript. LR prepared the manuscript. MW performed physical examination and clinical diagnosis of the patients. YS and LP performed the linkage studies. LP performed the statistical analysis. AMC provided oversight and conceptual guidance to the project, input into the functional significance of candidate genes, supervision of laboratory personnel, management of collaborations, preparation of the manuscript and all reporting requirements for granting agencies.

Rita M Cabral ${ }^{1,4}$, Mazen Kurban ${ }^{1,4}$, Lisa Rothman ${ }^{1}$, Muhammad Wajid ${ }^{1}$, Yutaka Shimomura $^{1}$, Lynn Petukhova ${ }^{1,2}$ and Angela M Christiano ${ }^{1,3}$

${ }^{1}$ Department of Dermatology, Columbia University, New York, NY, USA;

${ }^{2}$ Department of Epidemiology, Columbia University, New York, NY, USA;

${ }^{3}$ Department of Genetics and Development, Columbia University, New York, NY, USA and ${ }^{4}$ These authors contributed equally to this work., E-mail: amc65@columbia.edu

1 Urzua, B., Ortega-Pinto, A., Morales-Bozo, I., Rojas-Alcayaga, G. \& Cifuentes, V. Defining a new candidate gene for amelogenesis imperfecta: from molecular genetics to biochemistry. Biochem. Genet. 49, 104-121 (2011).

2 O'Sullivan, J., Bitu, C. C., Daly, S. B., Urguhart, J. E., Barron, M. J., Bhaskar, S. S. et al. Whole-Exome sequencing identifies FAM2OA mutations as a cause of amelogenesis imperfecta and gingival hyperplasia syndrome. Am. J. Hum. Genet. 88, 616-620 (2011).

3 Cho, S. H., Seymen, F., Lee, K. E., Lee, S. K., Kweon, Y. S., Kim, K. J. et al. Novel FAM20A mutations in hypoplastic amelogenesis imperfecta. Hum. Mutat. 33, 91-94 (2012).

4 Nalbant, D., Youn, H., Nalbant, S. I., Sharma, S., Cobos, E., Beale, E. G. et al. FAM20: an evolutionarily conserved family of secreted proteins expressed in hematopoietic cells. BMC Genomics 6, 11 (2005).

5 Simpson, M. A., Hsu, R., Keir, L. S., Hao, J., Sivapalan, G., Ernst, L. M. et al. Mutations in FAM20C are associated with lethal osteosclerotic bone dysplasia (Raine syndrome), highlighting a crucial molecule in bone development. Am. J. Hum. Genet. 81, 906-912 (2007).

6 Vogel, P., Hansen, G. M., Read, R. W., Vance, R. B., Thiel, M., Liu, J. et al. Amelogenesis imperfecta and other biomineralization defects in Fam20a and Fam20c null mice. Vet. Pathol. 6, 998-1017 (2012). 